(Aus dem physiologischen Institut der Universität Leipzig.)

\title{
Über \\ die Anordnung der homogenen Lichter auf der Mischlinie des Rotgrünblinden mit unverkürztem spektrum.
}

Von

E. Th. von Brüeke und N. Inonye.

(Mit 6 Textfiguren.)

Die Tatsache, dass objëktiv ganz verschieden zusammengesetzte Lichter gleichen physiologischen Reizwert - gleiche optische Valenz haben können, führte bekanntlich zu der Vorstellung, dass die optische Valenz der homogenen Lichter als eine komplexe Grösse aufzufassen sei, und dass sie sich aus mehreren Elementen, den sogenannten "Urvalenzen" zusammengesetzt denken lässt ${ }^{1}$ ).

Auf Grund der Lichtmischungsergebnisse nimmt bekanntlich sowohl die Hering'sche als die Young-Helmholtz'sche Farbentheorie an, dass der Reizwert jedes homogenen Lichtes sich für den Farbentüchtigen aus drei, für den partiell Farbenblinden aus zwei Urvalenzen zusammensetze. Ein fundamentaler Unterschied beider Theorien besteht aber darin, dass nach Hering für den Farbentüchtigen zwei, für den Farbenblinden eine dieser Urvalenzen sowohl positive als negative Werte in dem Sinne haben kann, dass aus der Mischung eines Lichtes mit positivem und eines Lichtes mit negativem Werte derselben Urvalenz ein Licht entsteht, in welchem der Wert dieser Urvalenz die algebraische Summe des positiven und negativen Wertes der Komponenten der Mischung ist. Nach der YoungHel m h ol tz' schen Theorie würde es sich dagegen nur um Urvalenzen mit positiven Werten handeln.

1) Vgl. E. Hering, Über Newton's Gesetz der Farbenmischung. Lotos, N. F., Bd. 7 S. 177 (206 ff.). 
Die Empfindungsqualitäten (Sehqualitäten), welche den einzelnen Urvalenzen entsprechen, sind in der Hering'sehen Theorie auf Grund einer psychologischen Analyse der Gesichtserupfindungen von vornherein bestimmt: der nur mit positiven Werten vorkommenden Urvalenz entspricht das Weiss, den beiden anderen, je nachdem sie positiven oder negativen Wert haben, ein ganz bestimmtes Gelb (Urgelb), bzw. Rot (Urrot) oder ein ganz bestimmtes Blau (Urblau) bzw. Grün (Urgrün). Die Theorie von Young-Helmboltz enthält an und für sich keine Bestimmung der Empfindungsqualitäten, welche den drei Urvalenzen zu entsprechen hätten, sondern fordert nur, dass keine zwei dieser drei Empfindungsqualitäten, der "Grundfarben", unter sich "komplementär" sind; meist wurde ein Rot, ein Grün und ein Blau oder Violett als die Grundfarben angenommen.

Eine gewisse Analogie bieten diese beiden Farbentheorien in der Deutung der Empfindungen der partiell Farbenblinden wie gesagt insofern dar, als nach beiden ein beliebiges Licht für einen solchen Farbenblinden nur zwei Urvalenzen besitzen würde: für die beiden Gruppen der Rotgrünblinden nach Hering nur eine weisse und eine gelbe oder blaue Urvalenz, und nach der Young-Helmholtz'schen Theorie zwei der oben genannten drei Urvalenzen, wobei sich allerdings die Farbenblinden der beiden Kategorien (der nach Hering Rotgrünblinden), im Gegensatze zur Auffassung dieses Forschers dadurch wesentlich voneinander unterscheiden würden, dass den einen die der erwähnten Grünempfindung entsprechenden physiologischen Vorgänge fehlten, den anderen die der erwähnten Rotempfindung entsprechenden. Wir können also nach beiden Theorien jedes Licht für den partiell Farbenblinden als ein Gemisch aus zwei Urvalenzen auffassen.

Nach den grundlegenden Auseinandersetzungen Hering's über die geometrische Darstellung von Gemischsystemen ${ }^{1}$ ) lässt sich die Gesamtheit aller denkbarer Gemische zweier Mischelemente, deren Werte sowohl positiv als negativ sein können, derart auf zwei unbegrenzten Geraden als den "Mischlinien" angeorduet denken, dass jedem Punkt auf einer dieser beiden Geraden (als eines „Mischlinienpaares") ein anderes bestimmtes Gemisch entspricht.

Im hier vorliegenden Falle der Mischung von Urvalenzen, wo nur das eine Mischelement mit positiven und negativen Werten, 
Über die Anordnung der homogenen Lichter auf der Mischlinie etc. 575

das andere aber ausschliesslich mit positiven Werten in Betracht kommt, handelt es sich nur um einen begrenzten Teil der einen Geraden eines Mischlinienpaares.

Da im folgenden die Anordnung der homogenen Lichter auf einem solchen begrenzten Mischlinienteile vom Standpunkte der Hering'schen Theorie für den Farbenblinden besprochen werden soll, müssen wir zunächst kurz auf die lineare Anordnung positiver und negativer Werte eines einzelnen Mischelementes eingehen und sodann die allgemeinen Verhältnisse bei der Mischung zweier Mischelemente und die Anordnung solcher Gemische auf einer Mischlinie erörtern, wobei wir uns zum Teil wörtlich an die Auseinandersetzungen Hering's halten wollen.

„Auf der unbegrenzten Geraden $m m$ (Fig. 1,2 und 3) möge jeder Punkt einem bestimmten, durch die ihm entsprechende Ordinate der unbegrenzten Geraden $l l$ dargestellten Wert eines beliebigen Etwas (E) bedeuten. Dieser Wert, welcher sowohl positiv als negativ sein kann, möge der E-Wert des bezüglichen Punktes und die Gerade $l l$ die Linie der E-Werte oder die E-Wertlinie heissen."

Den Schnittpunkt der E-Wertlinie mit der Geraden $m m$ bezeichnen wir als den Nullpunkt der E-Werte; alle Punkte der Linie $m m$, die links von diesem Nullpunkt liegen, mögen negativen E-Werten entsprechen, alle, die rechts von ihm liegen, positiven.

"Bezeichnen wir die zu den Punkten $A, C$ und $B$ (Fig. 1, 2 und 3) gehörigen Ordinaten oder $\mathrm{F}$-Werte mit $\alpha, \gamma, \beta$, die Strecke $A C$ mit $b$, die Strecke $C B$ mit $a$, so ist

$$
\begin{aligned}
& (\beta-\gamma):(\gamma-\alpha)=a: b \\
& (a+b) \gamma=a \alpha+b \beta^{\prime \prime} . \quad . \quad . \quad . \quad . \quad 1 .
\end{aligned}
$$

In dieser allgemeinen Gleichung ist stets das Vorzeichen der einzelnen E-Werte zu berücksichtigen; liegt also z. B. der Punkt $A$ links vom Nullpunkt der E-Werte, so ist $\alpha$ negativ in Rechnung zu bringen.

"Auf Grund der Gleichung 1. oder dureh Konstruktion lässt sich also, wenn für zwei Punkte der Geraden $m m$ die E-Werte gegeben sind, für jeden beliebigen dritten $\mathrm{E}$-Wert der zugehörige Punkt und für jeden beliebigen dritten Punkt der zugehörige E-Wert finden. Der zu einem beliebigen Punkte der Geraden mm gehörige E-Wert kann unter Benutzung eines in der physiologischen Optik bereits eingebürgerten Begriffes auch als die $\mathrm{Ma}$ asseinheit jenes Punktes bezeichnet werden." 
Wir denken uns nun zu den beiden Punkten $A$ und $B$ der Geraden $m m$ (Fig. 1, 2 und 3), deren E-Wertlinie $l l$ gegeben ist,

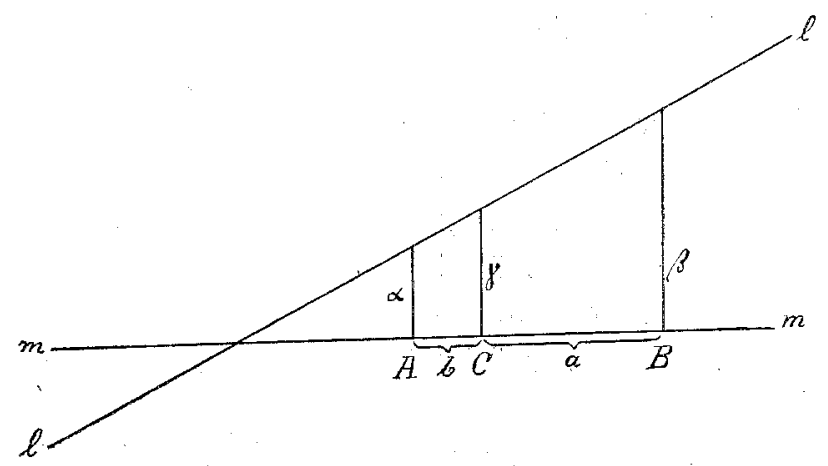

Fig. 1.

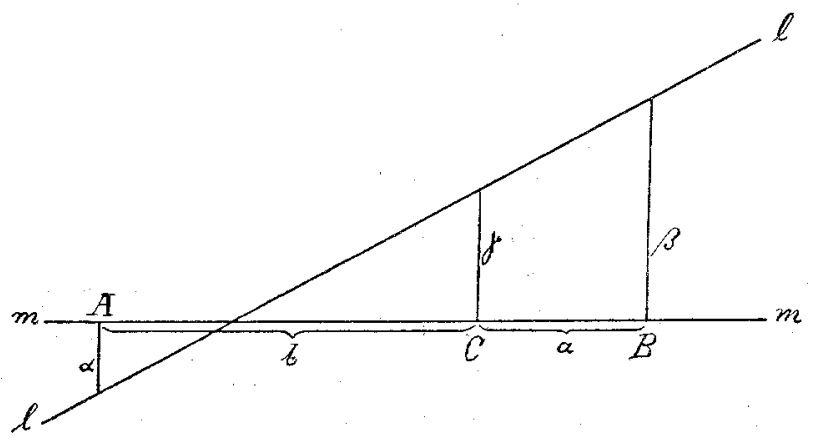

Fig. 2.

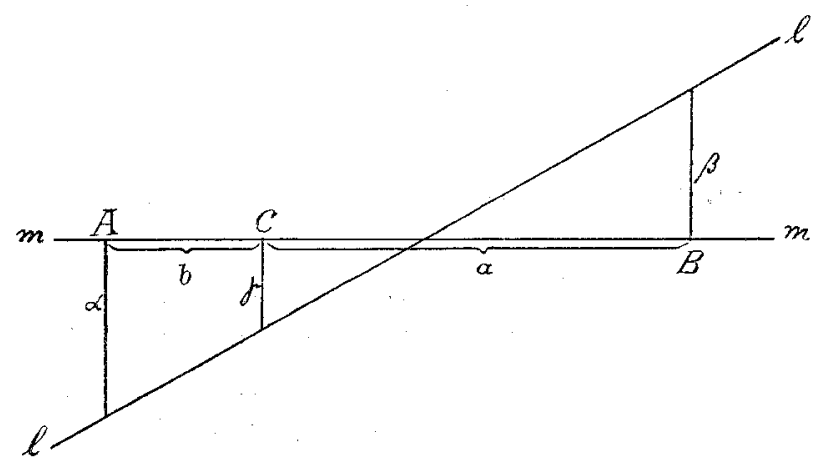

Fig. 3.

zwei beliebig grosse positive E-Quantitäten A und B und bestimmen, wie oft in der E-Quantität A der dem Punkte $A$ entsprechende 
Über die Anordnung der homogenen Lichter auf der Mischlinie etc. 577

E-Wert $\alpha$ enthalten ist, und wie oft in der E-Quantität $\mathrm{B}$ der dem Punkte $B$ entsprechende E-Wert $\beta$, d. h. wir messen A mit der Maasseinheit $\alpha$ und B mit der Maasseinheit $\beta$. Die so gefundenen Werte nennen wir $a$ und $b$.

„Denken wir uns nun die als schwerlos angenommene Gerade mm in den Punkt $A$ und $B$ mit zwei Gewichten belastet, welche sich zueinander verhalten wie die gefundenen Maasszahlen $a$ und $b$, und suchen den Schwerpunkt der so belasteten Geraden, so finden wir ihn im Punkte $C$, welcher die Strecke $A B$ derart teilt, dass das dem Punkte $A$ anliegende Teilstück $A C$ sich zu dem an $B$ grenzenden Teilstücke $B C$ verhält wie $b: a$, also

$$
A C: B C=b: \dot{a}{ }^{\prime \prime}
$$

Dividieren wir die Summe der beiden E-Quantitäten $A$ and $B$ durch die Summe der Maasszahlen $a$ und $b$, so erhalten wir den zum Punkte $C$ gehörigen E-Wert oder seine Maasseinheit; denn es gilt auch hier wieder die Gleichung 1. $(a+b) \gamma=b \beta-a \alpha$ und da $a \alpha=\mathrm{A}$ und $b \beta=\mathrm{B}$, so ist

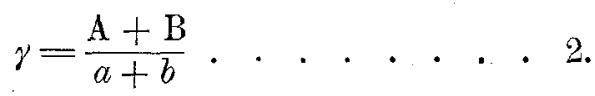

Zur binären Mischlinie wird eine Wertlinie dann, wenn jedem ihrer Punkte nicht nur ein bestimmter Wert ein es beliebigen Etwas entspricht, sondern je ein bestimmter Wert $\mathrm{zweier}$ in bezug auf irgendeine quantitative Eigenschaft vergleichbarer "Dinge".

Wie oben erwähnt wurde, kommt für uns nur ein begrenzter Teil einer solchen binären Mischlinie in Betracht, auf dem bestimmte Gemische aus einem mit positiven und negativen Werten auftretenden Mischelement $E_{1}$ und einem anderen nur mit positiven Werten auftretenden Mischelement $\mathrm{E}_{2}$ vertreten sind.

Zur Veranschaulichung einer solchen "Mischstrecke". möge Fig. 4 dienen: Jedem Punkt der Strecke $M M$ entspricht ein positiver oder negativer $\mathrm{E}_{1}$-Wert (die $E_{1}$-Wertlinie wird durch $L_{1} L_{1}$ angegeben) und ein positiver $\mathrm{E}_{2}$-Wert ( $L_{2} L_{2}$ entspricht der $\mathrm{E}_{2}$-Wertlinie).

„Jedem Punkt der Strecke $M M$ entspricht nunmehr ein anderes Gemisch von $E_{1}$ und $E_{2}$, an welchen wir $z u$ unterscheiden haben: erstens die Qualität oder das Mischungsverhältnis, welches gleich dem Verhältnis der beiden, dem Punkte zugehörigen Ordinaten ist; und zweitens die Quantität oder den Wert des Gemisches, welcher bestimmt ist durch die Summe der beiden zugehörigen Ordinaten und als Maasseinbeit des Gemisches bezeichnet werden soll." 
„Denkt man sich zwei beliebige, auf einer Mischlinie liegende Gemische (A und B) in einem beliebigen Mengenverbältnisse wieder miteinander gemischt, so erhält man stets ein Gemisch $\mathrm{C}$, welches bereits auf der Mischlinie vertreten ist und auf derselben $\mathrm{zwischen}$ den beiden Gemischen (A und B) gelegen ist."

Verhält sich die Zahl der zur Bildung des Gemisches $\mathrm{C}$ verwendeten Maasseinheiten des Gemisches A zur Zahl der verwendeten Maasseinheiten des Gemisches $\mathrm{B}$, wie $a$ zu $b$, und denken wir uns

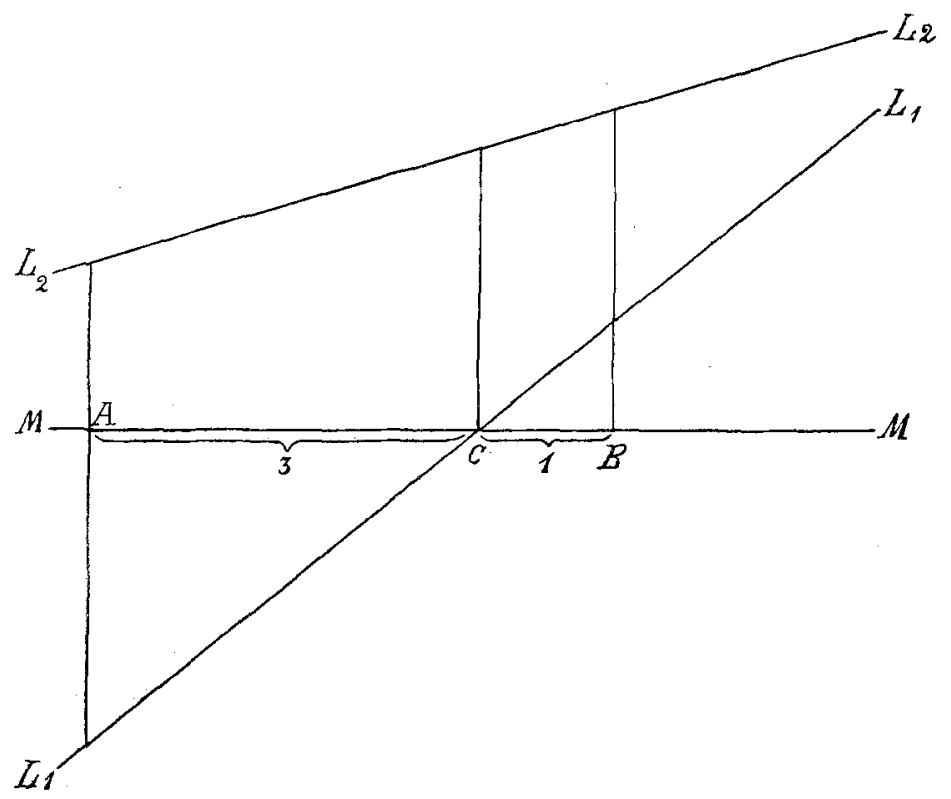

Fig. 4.

dementsprechend die Strecke $A B$ in den Punkten $A$ und $B$ mit Gewichten belastet, die sich umgekehrt proportional den Maasszahlen $a$ und $b$ verhalten, so ist der Schwerpunkt der so belasteten Strecke der Ort des Gemisches C.

Dieses Gesetz ermöglicht es uns auch dann den Ort eines aus zwei Gemischen gebildeten dritten Gemisches auf der Mischlinie zu finden, wenn uns das Verhältnis, in dem die einzelnen Gemische sich aus ibren Mischelementen zusammeusetzen, unbekannt bleibt.

Wenn uns andererseits der Ort eines bestimmten Gemisches A auf der Mischlinie bekannt ist, und ferner auch der Ort eines Gemisches $\mathrm{C}$ auf der Mischlinie gegeben ist, welches durch Mischung des Gemisches A und eines dritten Gemisches B, dessen Ort be- 
Über die Anordnung der homogenen Lichter auf der Mischlinie etc. 579

stimmt werden soll, erzeugbar ist, so können wir diesen Ort auf der Mischlinie in der Weise finden, dass wir wieder nach der Schwerpunktskonstruktion die fragliche Strecke $C B$ so lange wählen, dass sie sich zur bekannten Strecke $C A$ verhält wie die Anzahl der verwendeten Maasseinheiten des Gemisches A zur Differenz zwischen der bei der Mischung erzielten Maasseinheitenzahl des Gemisches C. und der Zahl der zur Mischung verwendeten Maasseinheiten des Gemisches A.

Um diese zuletzt erörterte Ortsbestimmung eines Gemisches auf einer Mischlinie an einem speziellen Beispiele zu erläutern, kehren wir zurück zu dem in Fig. 4 wiedergegebenen Abschnitt einer Mischlinie; und zwar nehmen wir an, dass uns der Ort des Gemisches A bei $A$ und der Ort des Gemisches $\mathrm{C}$ bei $C$ gegeben sei; der Ort des Gemisches $\mathrm{C}$ fällt mit dem Nullpunkt der $\mathrm{E}_{1}$-Wertlinie zusammen, der $\mathrm{E}_{1}$-Wert des diesem Punkte entsprechenden Gemisches ist demnach gleich Null, während sein $\dot{\mathrm{E}}_{2}$-Wert, wie der aller Gemische der Strecke $M M$ positiv ist. Nehmen wir nun an, wir bätten eine Maasseinheit des Gemisches A (mit seinem negativen $\mathrm{E}_{1}$-Wert) mit einer gewissen Menge eines uns betreffs seines Ortes und seiner Maasseinheit zunächst unbekannten Gemisches B gemischt und auf diese Weise vier Maasseinheiten des uns bekannten Gemisches $\mathrm{C}$ erhalten. Dann fänden wir den Ort jenes Gemisches $B$ in der Weise, dass wir der Strecke $C B$ eine solche Länge gäben, dass sich $C B$ zu $C A$ verhält, so wie $1:(4-1)$, also wie $1: 3$. Der dem Gemische A entsprechende Punkt der Mischlinie liegt demnach dreimal so weit von $C$ entfernt wie der dem Gemische $B$ entsprechende.

Gleichzeitig lernen wir auf diesem Wege auch die Maasseinheit des fraglichen Gemisches B kennen: Da nämlich die Anzahl der Maasseinheiten eines Gemisches stets gleich ist der Summe der zur Herstellung dieses Gemisches benutzten Maasseinheiten seiner Mischelemente, so müssen in unserem speziellen Falle bei der Herstellung der vier Maasseinheiten des Gemisches $\mathrm{C}$ ausser der einen Maasseinheit des Gemisches A noch drei Maasseinheiten des Gemisches B zur Verwendung gekommen sein. Eine Maasseinheit des Gemisehes B entspricht demnach einem Drittel jener Menge des Gemisches B, die wir in dem eben erörterten Falle zur Mischung verwendet haben.

Wenn wir nun die homogenen Lichter als für den Rotgrünblinden binäre Gemische auf einer derartigen "Mischstrecke" anordnen, so können wir als Endpunkte dieser Strecke einerseits ein 
relativ kurzwelliges Violett, andererseits ein spektrales Rot annehmen. Die Wahl dieser äussersten Lichter ist bis zu einem gewissen Grade willkürlich; doch ist darauf zu achten, dașs man speziell bei der Wahl des kurzwelligen Endlichtes iunerhalb jenes Spektralbereiches bleibt, in dem die einzelnen Lichter noch ohne merkliche Fluoreszenz der Augenmedien lebhafte Farbenempfindungen hervorrufen.

Wir können ferner die Maasseinheiten dieser beiden äussersten Lichter proportional jenen objektiven Stärken beider Lichter wählen, bei wëlchen die Misehung der beiden Lichter dern Farbenblinden farblo' erscheint, d. h. also so, dass bei gleichzeitiger Einwirkung je einer Maasseinheit beider Lichter weder eine Blau- noch eine Gelbempfindung resultiert, sondern nur die Weissvalenzen beider Lichter zup Geltung kommen. Nach den oben erörterten Prinzipien muss dann diê Wertlinie der positiven und negativen Blaugelbvalenzen unsere Misehstrecke genau in ihrem Mittelpunkt kreuzen, und an die Stelle dieses Nullpunktes der "Urvalenzlinie" muss das dem Rotgrünblinden neutrale Licht $\mathrm{N}$ zu liegen kommen.

Lassen wir nun den Rotgrünblinden aus je einer Maasseinheit (ME) des erwähnten langwelligen und kurzwelligen Lichtes ein für ihn farbloses (für den Farbentüchtigen purpurfarbenes) Gemisch herstellen, und lassen ihndann eime Gleichung zwischen diesem Gemisch einerseits und dẹm für ihn neutralen, Lichte $\mathrm{N}$ andererseits einstellen, so gelangen wir zu der Gleichung:

$1 \mathrm{MER}$ Rot +1.ME Violett $=2 \mathrm{ME} \mathrm{N}$.

Lassen wir ihn eine zweite Gleichung einstellen, und zwar zwischen den zwei Maasseinheiten von $\mathrm{N}$ einerseits und aus dem früher benutzten roten und etwa einem grünblauen Lichte andererseits, so werden wir sehen, dass er diesmal weniger Rot braucht als zuvor bei der "Ausgangsgleichung". Nehmen wir an, er brauchte 0,4 Maasseinheiten Rot, so wüssten wir, dass die von dem grünblauen Lichte verwendete Menge 1,6 Maassejnheiten dieses Lichtes entspricht. Ferner ergibt sich aus dem Verhältnis $0,4: 1,6$, dass der Ort des betreffenden grünblauen Lichtes auf der Mischlinie dem Orte des neutralen Lichtes viermal näher liegt als der Ort des roten Lichtes; wenn wir also der ganzen Mischlinie die Länge von $400 \mathrm{~mm}$ gegeben hätten und demnach die Distanz des gewählten Rot rom Lichte N $200 \mathrm{~mm}$ betrüge, so müsste der Punkt für das verwendete Grürblau $50 \mathrm{~mm}$ vom Orte des Lichtes $\mathrm{N}$ entfernt sein, weil

$$
50: 200=0,4: 1,6 \text {. }
$$


Über die Anordnung der homogenen Licbter auf der Mischlinie etc. 581

Nach dieser Methode lassen sich die Orte aller zwischen dem gewählten äussersten Rot und dem gewählten äussersten Violett gelegenen homogenen Lichter auf der Mischlinie bestimmen.

Ein anderer Weg, der anscheinend zu dem gleichen Ziele führen würde, wäre der folgende: Wir könnten dem Rotgrünblinden nacheinander Gleichungen einstellen lassen zwischen jenem Gemisch aus Rot und Violett einerseits und irgendeinem im Spektrum dazwischen liegenden homgenen Lichte andererseits, und könnten dann aus der Zahl der jeweilig verwendeten Masseinheiten des roten und violetten Lichtes die Maasseinheit und den Ort des dazwischenliegenden-tichtes auf der Mischlinie berechnen.

Bei Benützung dieser Methode würden sich aber Fehler in die Versuchsergebnisse einschleichen können. Die alte Annahme; nach welcher Farbengleichungen auch dann als solche fortbestehen, wenn die in ihnen enthaltenen Lichter in gleichem Verhältnis verstärlít oder abgeschwächt werden, wurde zwar seinerzeit. für das helladaptierte Auge von v. Kries und von Hering durch"besonders daraufgerichtete Versuche bestätigt, erwies sich aber weiterhin für mehr oder minder dunkeladaptierte Augen nicht gültig. In $\mathrm{Rück}$ sichthierauf untersuchten wir nur mit helladaptiertem Auge uud bestimmten überdies den Ort derspektralén Lichter auf der Mischlinie nur inder Weise, dass wir Gleichungen herstellten, bei denen die eine Hälfte des beobachteten Feldes bei allen Versuchen mit einer ganz konstanten Menge des für den untersuehten Farbenblinden neutralen Lichtes gefüllt war $(499,5 \lambda)$, während auf der anderen Feldhälfte bei der einen Serie von Versuchen Gemische aus einem bestimmten Rot $(631 \lambda)$ und verschiedenen kurzwelligen Lichtern eingestellt wurden, bei der anderen Versuchsserie Gemische aus einem bestimmten Violett (443 $\lambda$ ) und verschiedenen langwelligen Lichtern, so dass also nur Gleichungen hergestellt wurden, die fürden Rotgrünblinden farblos waren und auf der einen Seite aus dem nach Qualität und Quantität unveränderten Lichte von $499,5 \lambda$ bestanden.

Die Anordnung der homogenen Lichter auf einer nach dem erörterten Prinzipe konstruierten Mischlinie ergibt eine Einreihung der Lichter in der Weise, dass die zwèi Wertlinien, die den beiden für 
den Rotgrünblinden in Betracht kommenden Urvalenzen entsprechen, ger a de Linien sind. Hierbei ist der Winkel, den wir die BlaugelbWertlinie mit der Mischlinie einschliessen lassen, willkürlich wählbar, und auch die Lage und Neigung der Wertlinie der Weissvalenzen relativ zur Mischlinie lässt sich aus diesen Versuchen nicht ermitteln.

Zwischen unseren Versuchen und den sogenannten Eichungen eines Spektrums für die Rotgrünblinden mit Hilfe zweier den beiden Enden des Spektrums naheliegender Lichter, wie sie z. B. zuletzt von J. v. Kries und W. Nagel ${ }^{1}$ ) ausgeführt wurden, besteht keinerlei Analogie. In den Versuchen von v. Kries und Nagel handelte es sich darum, für die Rotgrünblinden zwischen den einzelnen Lichtern eines ganz bestimmten Spektrums (Gaslicht mit Triplexbrennern) einerseits und Gemischen aus einem kurzwelligen $(460,8 \lambda)$ und einem langwelligen $(645 \lambda)$ Lichte eines gleichen Spektrums andererseits Gleichungen herzustellen. Auf diese Weise lässt sich die "Verteilung der Rot- und der Blauwerte" für ein ganz bestimmtes Spektrum unter der Voraussetzung bestimmen, dass die Sättigungsdifferenzen, die der Rotgrünblinde im Spektrum zwischen den verschiedenen "gelbwertigen" und "blauwertigen" Farben wabrnimmt, bei Benutzung des stäbchenfreien fovealen Bezirkes auf eine gleichzeitige Erzeugung der beiden Grundempfindungen des Farbenblinden zurückzuführen seien. Während also derartige Eichungen immer nur die Verbältnisse für ein ganz bestimmtes Spektrum wiedergeben können, ist die Anordnung der homogenen Lichter auf einer Mischlinie, wie sie hier mitgeteilt wird, völlig unabhängig von der Dispersion des benutzten Spektrums und von der benutzten Lichtquelle. Der Ort eines Lichtes auf der Mischlinie wird durch eine Eigenschaft jenes Lichtes bestimmt, die der betreffenden Strahlung an und für sich - in Beziehung zu einem bestimmten Sehorgan unter allen Umständen zukommt. Anders steht es mit den Maasseinheiten, die nach den Ergebnissen der Gleichungen den einzelnen Lichtern zukommen; diese sind von der Energie der einzelnen Strahlungen in dem benutzten Spektrum abhängig, und da das Verhältnis der Energie der verschiedenen spektralen Lichter je nach ihrer Verteilung im Spektrum und je nach der benutzten Lichtquelle

1) J. v. Kries und W. Nagel, Über den Einfluss von Lichtstärke und Adaptation auf das Sehen des Dichromaten (Grünblinden). Zeitschr. f. Psych. u. Physiol. d. Sinnesorgane Bd. 12, S. 1. 1896. 
Über die Anordnung der homogenen Lichter auf der Mischlinie etc. 583

grossen Verschiedenheiten unterworfen ist, so muss auch das Verbältnis der Maasseinheiten der einzelnen Lichter bei Spektren verschiedener Herkunft verschieden sein. Während also der Ort eines Lichtes auf der Mischlinie von der Art des benutzten Spektrums ganz unabhängig ist, hat die $\mathrm{Ma}$ asseinheit dieses Lichtes nur in bezug auf das benutzte Spektrum eine Bedeutung.

Wir führten unsere Versuche an dem grossen Hering'schen Spektralapparat aus, bei dem uns als Lichtquellen Nernstlampen mit je drei parallelstehenden Glühstäben dienten. Da die Schwankungen des Stadtstromes eine exakte, längere Zeit dauernde quantitative Untersuchung ausserordentlich erschweren, speisten wir diese Nernstlampen mit einer eigenen Akkumulatorenbatterie von 50 grossen Zellen, die täglich friseh geladen wurden. Die Abnahme der Stromstärke während der Dauer einer Versuchsreihe (2-3 Stunden) war so geringfügig, dass wir während dieser Zeit keine Änderung der hergestellten Gleichungen beobachten konnten.

Die Grösse des beobachteten Feldes entsprach $30 \mathrm{~mm}$ in $336 \mathrm{~mm}$ Distanz vom Auge, also betrug seine Winkelgrösse etwa $5^{\circ}$ und die Grösse des Netzhautbildes etwa $1,3 \mathrm{~mm}$. Um den Einfluss schwankender Adaptationszustände des beobachtenden Auges möglichst auszuschliessen, wurden die Versuche stets in den Vormittagsstunden ausgeführt, und zwar wurde während jedes Versuches in Intervallen von etwa 10 Minuten mittelst eines Polariphotometers die Lichtstärke der weissgetünchten Zimmerwand gemessen und durch partielles Herablassen oder Hochziehen der undurchsichtigen Fensterrouleaus immer wieder auf einen bestimmten Wert eingestellt, so dass das Auge, dessen Blick zwischen je zwei Beobachtungen über die betreffende Wand wanderte, stets annähernd den gleichen Adaptationszustand bewahrte.

Als Versuchsperson diente ausschliesslich der eine von uns (Brücke,) der selbst dem Typus der Rotgrünblinden mit unverkürztem Spektrum angehört.

Der benutzte Apparat enthält drei Spektralapparate (I, II und III) ohne Okularlinsen; jeder Kollimator lieferte eines der drei zur Herstellung einer Gleichung nötigen Lichter. Durch einen im folgenden als Vorderspalt (I, II und III) bezeichneten Spalt wird aus jedem der drei Spektren (I, II und III) ein schmaler Streifen ausgeschnitten, 
dessen Licht dann zur Herstellung der Gleichung benutzt wird. Die Menge dieses Lichtes ist also proportional einerseits der Breite des Kollimatorspaltes, andererseit der Breite des Vorderspaltes.

AIs "Ausgangsgleichung" diente bei all unseren Versuchen die Gleichung

Rot $(631 \lambda)+$ Violett $(443 \lambda)=\operatorname{Grün}(499,5 \lambda)$,

und zwar gaben wir willkürlich der unter allen Umständen konstant gehaltenen Grünmenge [50 Teilstriche der Schraube des Kollimatorspaltes $(=0,5 \mathrm{~mm}) \times 50$ Teilstriche der Schraube des Vorderspaltes $(=0,5 \mathrm{~mm})$ den Wert von zwei Maasseinheiten Grün und der jeweilig zur Gleichung nötigen Menge des Rot und des Violett den Wert je einer Maasseinheit. Wir könnten diese Gleichung also auch so schreiben:

$1 \mathrm{ME} 631 \lambda+1 \mathrm{ME} 443 \lambda=2 \mathrm{ME} 499,5 \lambda$.

Obgleich alle Faktoren, die wir regeln konnten, konstant gehalten wurden, schwankten die zur Einstellung dieser Ausgangsgleichung nötigen Violett- und Rotmengen an den einzelnen Versuchstagen doch innerhalb nicht unbeträchtlicher Grenzen: Bei den Dezemberversuchen, welche die Bestimmung des Ortes der $\mathrm{kurz}-$ welligen Lichter betrafen, erhielten wir an den einzelnen Versuchstagen für die Lichter yon $443 \lambda$ und $631 \lambda$ jene Maasseinheiten, die im ersten und zweiten Horizontalstabe der beistehenden Tabelle I. angegeben sind; bei den Februarversuchen, die zur Bestimmung des Ortes der langwelligen Lichter angestellt wurden, erhielten wir jene Werte, die im dritten und vierten Horizontalstabe der Tabelle stehen:

Tabelle 1 .

\begin{tabular}{|c|c|c|c|c|c|c|c|c|c|c|}
\hline $\begin{array}{c}\text { 1. }-16 . \text { Dez. } \\
1910\end{array}$ & $\left\{\begin{array}{l}\mathrm{MF} \text { v. } 443 \lambda \\
\mathrm{ME} \text { v. } 631 \lambda\end{array}\right.$ & $\begin{array}{r}1610 \\
412\end{array}$ & $\begin{array}{r}1760 \\
464\end{array}$ & $\begin{array}{r}1683 \\
444\end{array}$ & $\begin{array}{r}1575 \\
450\end{array}$ & $\begin{array}{r}1748 \\
484\end{array}$ & $\begin{array}{r}1766 \\
440\end{array}$ & $\begin{array}{r}1861 \\
480\end{array}$ & $\begin{array}{r}1744 \\
466\end{array}$ & $\begin{array}{r}1718 \\
455\end{array}$ \\
\hline $\begin{array}{c}\text { 5.-28. Febr. } \\
1911\end{array}$ & $\left\{\begin{array}{l}\text { ME v. } 443 \lambda \\
M E \text { v. } 631 \lambda\end{array}\right.$ & $\left|\begin{array}{r}2476 \\
480\end{array}\right|$ & $\begin{array}{r}2411 \\
480\end{array}$ & $\begin{array}{r}2402 \\
476\end{array}$ & $\begin{array}{r}2417 \\
468\end{array}$ & $\begin{array}{r}2425 \\
460\end{array}$ & $\begin{array}{r}2435 \\
480\end{array} \mid$ & $\left|\begin{array}{r}2428 \\
474\end{array}\right|$ & - & - \\
\hline
\end{tabular}

Fs zeigt sich, dass die an aufeinanderfolgenden Tagen für die Lichter von $443 \lambda$ und $631 \lambda$ erhaltenen Maasseinheiten relativ gut übereinstimmen; dagegen weichen die Maasseinheiten, die im Dezember beobachtet wurden, wesentlich von den im Februar gefundenen $a b$, was wohl auf eine mit der Zeit eingetretene Änderung der Nernstlampen zurückzuführen seia dürfte. 
Da nach dem früher Erörterten für die Bestimmung des Ortes eines spektralen Lichtes auf der Mischlinie die Maasseinheiten der als Endpunkte der Linie gewählten Lichter die bestimmende Rolle spielen, haben wir an jedem Versuchstage vor Beginn der einzelnen Versuchsreihe zunächst unsere Ausgangsgleichung fünf- oder zehnmal eingestellt und das Mittel der bei diesen Gleichungen gefundenen Maasseinheiten für $443 \lambda$ und $631 \lambda$ als "jeweilige Maasseinheit" für die Versüche des betreffenden Tages gewählt und nach diesen Einheiten die ,jeweiligen Orte" der einzelnen Lichter auf der Mischlinie bestimmt. Nach Abschluss aller unserer Versuche haben wir auch die durchschnittlich aus sämtlichen Versuchen sich ergebende Maasseinheit für die beiden Endlichter berechnet und die Orte der übrigen homogenen Lichter dann auf Grund dieser mittleren Maasseinheiten umgerechnet. Die auf diese Weise gefundenen "mittleren Orte" der verschiedenen Lichter weichen in den meisten Fällen gar nicht, in den anderen nur sehr wenig von jenen Orten ab, die sich als Mittelwert aus den ;jeweiligen Orten" ergaben.

Es sei nun an einem Beispiel der Gang eines Versuches erläutert:

\section{Versuch rom 28. Februar 1911.}

Es wưde zunächst für Brücke die Gleichung $631 \lambda+443 \lambda=499,5 \lambda$ eingestellt. Kollimator $I=68^{17 / 20}$ Skalenteile $=631 \lambda$; Kollimatorspalt $=40$ $(=0,4 \mathrm{~mm}) ;$ Kollimator II $=75^{13} / 20$ Skalenteile $=443 \lambda ;$ Kollimatorspalt $=50$ $(=0,5 \mathrm{~mm}) ;$ Kollimator III $=39^{12 / 20}$ Skalenteile $=499,5 \lambda ;$ Kollimatorspalt $=50$ $(=0,5 \mathrm{~mm})$ Vorderspalt $=50 .(=0,5 \mathrm{~mm})$.

Die Kollimatorspalten und der Vorderspalt des Kollimators III blieben, wie bei allen Versuchen, konstant und die Gleichung wurde nur durch Variation der Vorderspalten I und II eingestellt. Bei fünf Einstellungen betrugen die Spaltbreiten in Hunderstel-Millimeter ausgedrückt folgende Werte:

Vorderspalt I: $12,0,12,7,12,0,11,7,11,5$. Mittel : 12,0 .

Vorderspalt.II: $46,6,48,6,50,5,47,2,50,7$. Mittel: 48,7 .

Die Maasseinheit für $631 \lambda$ betrug demnach $40 \times 12,0=480$, jene für $443 \lambda$ betrug $50 \times 48,7=2485$.

Es bestand also die Gleichung: 480 Teile $631 \lambda+2435$ Teile $443 \lambda=2500$ Teile $499,5 \lambda$.

Sodann wurden die eigentlichen Versuche mit verschiedenen langwelligen Lichtern begonnen. Die Kollimatoren II und III änderten ihre Stellung nicht, aber im Kollimator I wurde zunächst anstatt $631 \lambda$ das Licht von $550 \lambda$ eingestellt; auch wurde der Kollimatorspalt I von 40 auf 50 Skalenteile erweitert. Nunmehr stellte Brücke fünf Gleichungen ein: $550 \lambda+443 \lambda=499,5 \lambda$. 
Bei diesen fünf Einstellungen ergaben sich folgende Spaltbreiten für die Vorderspalten I und II:

Vorderspalt I: 11,7, 11,7, 11,5, 11,0, 11,2. Mittel: 11,4.

Vorderspalt II : $45,1,46,6,43,3,46,0,46,2$. Mittel : 45,4 .

Es bestand also die Gleichung: $570(50 \times 11,4)$ Teile $550 \lambda+2270(50+45,4)$ Teile $443 \lambda=2500$ Teile $499,5 \lambda$.

- Die Berechnung des Ortes des Lichtes von $550 \lambda$ und seiner Maasseinheit geht nun folgenden Gang:

Die bei der letzten Gleichung verwendeten 2270 Teile von $443 \lambda$ entsprechen $\frac{2270}{2435}$ Maasseinheiten $=0,93$ ME.

Da die 2500 Teile 499,5 $\lambda$ zwei Maasseinheiten entsprechen, können wir statt der letaten Gleichung auch schreiben:

$$
(2-0,93) \mathrm{ME} 550 \lambda+0,93 \mathrm{ME} 443 \lambda=2 \mathrm{ME} 499,5 \lambda \text {. }
$$

Die verwendete Menge von $550 \lambda$ ( 570 Teile) entsprach also 1,07 Maasseinheiten, so dass sich hieraus die Maasseinheit des Lichtes von $550 \lambda$ berechnet zu $\frac{570}{1,07}=533$.

Zur Bestimmung des Ortes dieses Lichtes dient folgende Überlegung: Wählen wir z. B. als Länge der Mischlinie, d. h. als Distanz der äussersten berücksichtigten Lichter (631 $\lambda$ und $443 \lambda$ ) die Länge ron $400 \mathrm{~mm}$, dann liegt das neutrale Grün $(499,5 \lambda)$ im Halbierungspunkt dieser Strecke nach der Gleichung

$1 \mathrm{ME} 631 \lambda+1 \mathrm{ME} 443 \lambda=2 \operatorname{ME} 499,5 \lambda$.

Für das Licht von $550 \lambda$ galt die Gleichung:

$1,07 \mathrm{ME} 550 \lambda+.0,93 \mathrm{ME} 443 \lambda=2 \mathrm{ME} 499,5 \lambda$.

Wir müssen demnach das Licht 550 so auf der Mischlinie eintragen, dass seine Distanz von dem neutralen Lichte sich zur Distanz des Lichtes $443 \lambda$ von diesem neutralen Lichte verhält wie $0,93: 1,07$. Da die Distanz $443 \lambda \longleftrightarrow \longrightarrow$ $499,5 \lambda$ auf der Mischlinie $200 \mathrm{~mm}$ beträgt, so ergibt die Rechnung:

$$
\mathrm{X}: 200=0,98: 1,07
$$

als Distanz des Lichtes $550 \lambda$ von dem neutralen Grün die Strecke von $174 \mathrm{~mm}$.

In dieser Weise wurde an jedem Versuchstage aus je fünf Gleichungen der Ort und die Maasseinheit für durchschnittlich sechs Lichter berechnet.

Obwohl diese Gleichungen unter den genannten Kautelen mit grösster Sorgfalt eingestellt und vor der Protokollierung immer mehrmals nach Pausen kontrolliert wurden, weichen die an den einzelnen Versuchstagen erhaltenen Werte nicht unbeträchtlich von einander ab. Für die ,jeweiligen " ${ }^{1}$ ) Orte ergeben sich als mittlere Fehlerbreite $11,6 \%$, das bedeutet auf unserer Mischlinie von $400 \mathrm{~mm}$ Länge eine mittlere Fehlerbreite in der Lage der Orte von etwa

1) Vgl. S. 585. 
Über die Anordnung der homogenen Lichter auf der Mischlinie ete. 587

$23 \mathrm{~mm}$. Die Fehler waren für die kurzwellige Hälfte des Spektrums (also bei den Gleichungen $631 \lambda$ + kurzwelliges Licht $=499,5 \lambda$ ) kleiner als für die langwellige Hälfte, ohne dass sich hierfür ein Grund angeben liesse. Eine ähnliche Fehlerbreite ergab sich auch für die jeweiligen Maasseinheiten der einzelnen Lichter.

In der nebenstehenden Tabelle sind die Mittelwerte der jeweiligen Orte der spektralen Lichter angegeben, und zwar ausgedrückt durch ihre Distanz von der Mitte der $400 \mathrm{~mm}$ langen Mischlinie, also von dem neutralen Grün; die Maasseinheiten sind als Produkte der Kollimatorspaltbreite $\times$ Vorderspaltbreite aufzufassen; sie haben kein allgemeines Interesse und seien nur der Vollständigkeit wegen angegeben.

In Fig. 5 ist die auf Grund dieser Ortsangaben gezeichnete Mischlinie in verkleinertem Maassstabe wiedergegeben. Obwohl die einzelnen Lichter, deren Orte auf der Mischlinie bestimmt wurden, nicht um gleiche Wellenlängendifferenzen auseinanderliegen, so lässt diese Mischlinie doch erkennen, dass, entsprechend der raschen Änderung der Farbensättigung, die der Rotgrünblinde in der Umgebung des neutralen Punktes des Spektrums wahrnimmt, die Distanzen der dem neutralen Grün benachbarten Lichter auf der

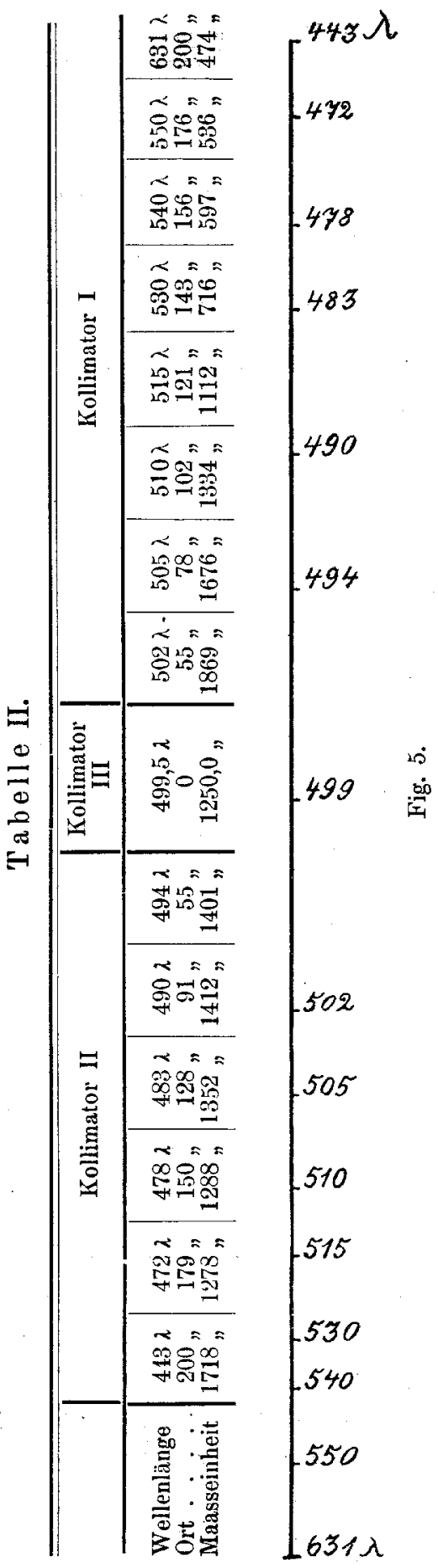


Mischlinie im Vergleich zu ihrer Wellenlängendifferenz auffallend gross sind, und dass die entsprechenden Distanzen um so kleiner werden, je mehr sich die Lichter einem Ende des Spektrums nähern. Eine kleine Abweichung hiervon zeigen nur die Distanzen der Lichter $530 \lambda, 540 \lambda$ und $550 \lambda$, da in diesem Falle der Ort des Lichtes von $540 \lambda$ weiter gegen das Ende der Mischlinie zu liegen sollte; offenbar ist diese Abweichung nicht physiologisch bedingt, sondern als Fehler anzusehen.

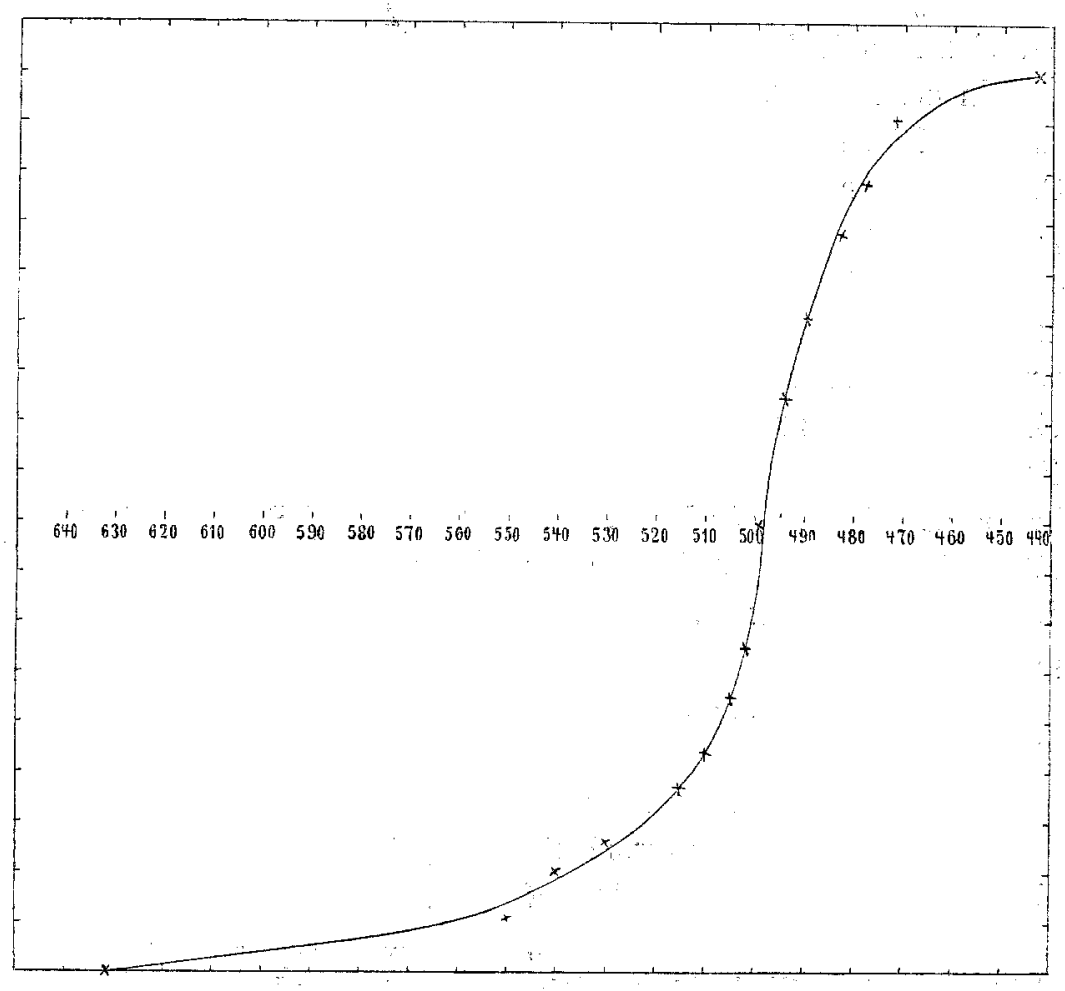

Fig. 6.

Entsprechend den in der Einleitung erörterten Prinzipien ist diese Mischlinie so konstruiert, dass jeder ibrer Punkte einem bestimmten Gemisch aus den Mischelementen: der Blauvalenz und der Weissvalenz, oder der Gelbvalenz und der Weissvalenz entspricht; und zwar sind die spektralen Lichter als Gemische so auf die Mischlinie gesetzt, dass die Mengen der den Maasseinheiten der einzelnen Gemische zukommenden Blau- bzw. Gelbwerte und Weisswerte linear wachsen bzw. abnehmen. Es schien uns nicht uninteressant, 
Über die Anordnung der homogenen Lichter auf der Mischlinie etc. 589

jene Kurve zu zeichnen, welche resultiert, wenn wir die den einzelnen Lichtern zukommenden Maasseinheiten ihrer farbigen Valenzen als Ordinaten über den betreffenden Lichtern in ein mit homogener Dispersion angenommenes Spektrum einzeichnen. Auf der Misehlinie sind die Maasseinheiten der farbigen Valenzen der einzelnen Lichter dem Abstand der Lichter vom Nullpunkt der Blau-Gelbvalenzlinie proportional, so dass wir die gesuchte Kurve am einfachsten in der Weise erhalten, dass wir über den Lichtern eines Spektrums mit homogener Dispersion als Ordinaten die jeweiligen Abstände dieser Lichter von der Mitte der in Fig. 5 wiedergegebenen Mischlinie eintragen. Auf diese Weise ist die Kurve der Fig. 6 erhalten; die Asymmetrie dieser Kurve ist eine notwendige Folge der verschiedenen Art der Verteilung der gelb- und blauwertigen Lichter im Spektrum im Vergleich zu ihrer Verteilung auf der Urvalenzlinie. Im übrigen sehen wir, dass die Endpunkte der so aufgetragenen Ordinaten sich innerhalb einer gewissen Fehlergrenze einer regelmässigen Kurve ziemlich gut anschmiegen, woraus wir wohl auch schliessen dürfen, dass sich bei der von uns ermittelten Anordnung der hơmogenén Lichter auf einer Mischlinie kèine gröberen Irrtümer eingeschlichen haben.

Die Richtigkeit der hier ermittelten Verteilung der homogenen Lichter auf der Mischlinie lässt sich auch leicht durch eine grosse Zahl von Gleichungen kontrollieren, insoweit der oben erwähnte Übelstand der Inkonstanz der Gleichungen nicht in Betracht kommt. Wenn nämlich Ort und Maasseinheit verschiedener Lichter gegeben sind, so lässt sich hieraus berechnen, in welchem Verhältnis gemischt zwei beliebige Lichter sich zu einer Gleichung mit einem dritten Licht vereinigen lassen.

Es sei dies an folgendem speziellen Falle demonstriert:

Auf Grund der Maasseinheiten und der Orte auf der Mischlinie sollen für den Rotgrünblinden die für die Gleichung $631 \lambda+502 \lambda=515 \lambda$ nötigen Lichtmengen berechnet werden:

Die Distanz zwischen dem Lichte von $631 \lambda$ und jenem von $515 \lambda$ beträgt auf der Mischlinie von $400 \mathrm{~mm}$ Länge $79 \mathrm{~mm}$; der gegenseitige Abstand der Lichter $502 \lambda$ und $515 \lambda$ beträgt $66 \mathrm{~mm}$. Demnach müssen wir zu der Gleichung $631 \lambda+502 \lambda=515 \lambda$ den Mengen der Lichter von $631 \lambda$ und $502 \lambda$ das Verhältnis von $66: 79$ geben, d. h. die Mengen beider Lichter in dem Gexaisch müssen sich umgekehrt verhalten wie ihre Abstände von dem dazwischenliegenden Lichte, mit dem die Gleichung hergestellt werden soll. Wollen wir also zu der Gleichung eiue Maasseinheit $631 \lambda$ verwenden, so müssen wir sie mischen mit $1,20\left(=\frac{79}{66}\right)$ Maasseinheiten $502 \lambda$, und dies Gemisch muss dann gleich sein : 2,20 Maasseinheiten $515 \lambda$. 
590 E. Th. von Brücke und N. Inouye: Ủber die Anordnung etc.

Die Herstellung solcher Kontrollgleichungen wird dadurch etwas kompliziert, dass man die einzelnen Lichter aus technischen Gründen oft nicht in jenen Kollimatoren einstellen kann, für die ihre Maasseinheiten ursprünglich bestimmt worden waren, so dass man dann erst experimentell die Maasseinheit für den jeweilig zu verwendenden Kollimator bestimmen muss.

Wir haben auf diese Weise eine Reihe von Kontrollgleichungen berechnet und eingesteilt, die zu einem kaum erwartet günstigen Resultate führten, da sie zum Teil vollkommen stimmten, zum Teil durch geringfügige Variationen korrigiert werden konnten, woraus wir schliessen können, dass die oben erwähnte Inkonstanz der Gleichungen bei proportionalen Intensitätsänderungen der bezüglichen Lichter bei unseren Kontollversuchen ohne wesentlichen Einfluss auf das Ergebnis gewesen ist, und dass in der ermittelten Anordnung der spektralen Lichter auf der Mischlinie (abgesehen von der unvermeidlichen Fehlerbreite) keine gröberen Unrichtigkeiten bestehen.

\section{Zusammenfassung.}

Ausgehend von der Auffassung der homogenen Lichter als Urvalenzgemische nach Hering, werden in dèr vorliegenden Arbeit die homogenen Lichter auf Grund spektraler Gleichungen für den Rotgrünblinden so auf einer "Mischlinie" angeordnet, dass die eine Hälfte der Mischlinie die blauwertigen, die andere die gelbwertigen Lichter umfasst. Dabei ist die Grösse der den Lichtern entsprechenden bunten Valenzen (Grösse ihrer Blau- bzw. Gelbvalenz) eine lineare Funktion des Abstaudes der Lichter von der Mitte der Mischlinie (dem neutralen Punkte des Spektrums), und die Weissvalenz der Lichter ist eine lineare Funktion ihres Abstandes von dem irgendwo in der Verlängerung der Mischlinie gelegenen Punkte, in welchem die Linie der Weissvalenzen die Mischlinie schneidet. 